\title{
Papel Atual da Angiotomografia de Coronárias na Avaliação Inicial da Doença Coronariana
}

\author{
Current Role of Coronary Angiotomography in the Initial \\ Evaluation of Coronary Disease
}

\author{
Joana Barreto Bittencourt ${ }^{1}$, Jorge Andion Torreão ${ }^{2}$, José Rocha Filho ${ }^{2}$ \\ ${ }^{1}$ Cardiologista estagiária em imagem cardiovascular; ${ }^{2}$ Serviço de Imagem do Hospital \\ Santa Izabel;Salvador, Bahia, Brasil
}

Correspondence addresses:

Dra. Joana Bittencourt

bittencourtjoana@yahoo.com

Received: January 17, 2019

Revised: February 25, 2019

Accepted: March 10, 2019

Published: March 27, 2019

Data Availability Statement: All relevant data are within the paper and its Supporting Information files.

Competing interests: The authors have declared that no competing interests exist.

\section{Copyright}

(C) 2019 by Santa Casa

de Misericórdia da Bahia.

All rights reserved.

ISSN: 2526-5563
A angitomografia (AngioTC) como estratégia de primeira linha para avaliar pacientes com dor torácica e probabilidade intermediária de doença arterial coronariana mostrou-se de extrema importância nos estudos revelados (ACCURACY, CONFIRM, SCOT HEART, PROMISE, CORE320, PACIFIC, NXT Trial). Esta técnica é não invasiva e permite o diagnóstico da doença coronariana no estágio inicial, com a identificação da placa rica em lipídio. O National Institute for Health and Care Excellences (NICE) Clinical Guideline, em 2016, traz como recomendação a realização da angitomografia de artérias coronárias como estratégia de primeira linha em todos os pacientes que apresentaram dor torácica com a suspeita de doença arterial coronariana. Desta forma, pode-se afirmar que a adição da angiotomografia à estratégia padrão reduz eventos coronarianos, principalmente infarto não fatal. Palavras-chave: Angiotomografia de coronárias; Doença arterial coronariana; Dor torácica.

The angiotomography (AngioTC) as a first-line strategy to evaluate patients with chest pain and intermediate probability of coronary artery disease was of extreme importance in the following studies: ACCURACY, CONFIRM, SCOT HEART, PROMISE, CORE320, PACIFIC, NXT Trial. This technique is noninvasive and allows the diagnosis of coronary disease in the initial stage, with the identification of the lipid-rich plaque. The National Institute for Health and Care Excellences (NICE) Clinical Guideline (2016) recommends coronary angiotomography as a first line strategy in all patients that presented chest pain with suspected coronary artery disease. So, the addition of angiotomography to the standard strategy reduces coronary events, mainly non-fatal infarction.

Keywords: Coronary angiotomography; Coronary artery disease; Thoracic pain.

\section{Introdução}

A abordagem de pacientes com suspeita de dor torácica por doença arterial coronariana é muito discutida na cardiologia. Muitos pacientes, principalmente de probabilidade baixa e intermediária, não são diagnosticados corretamente para doença arterial coronariana e aproximadamente um terço dos pacientes classificados com dor torácica não cardíaca evoluem com morte cardiovascular ou apresentam evento coronário agudo em um prazo de 5 anos. ${ }^{1}$ 
Nesse contexto, surge o papel da angiotomografia das artérias coronárias (AngioTC) como estratégia de primeira linha para avaliar pacientes com dor torácica e probabilidade intermediária de doença arterial coronariana (DAC) (Figura 1). ${ }^{1}$

\section{Identificação da Placa na Fase Inicial da Doença Coronariana}

Duas grandes utilidades da angiotomografia na investigação da dor torácica são a caracterização da placa e a quantificação da estenose (Figuras 2 e 3 ).

AAngioTC permite uma excelente visão, tanto luminal quanto parietal das coronárias, podendo identificar placas ateroscleróticas vulneráveis mesmo antes de qualquer repercussão hemodinâmica.

Motoyama e colaboradores ${ }^{2}$ publicaram, em 2015, no Journal of the American College of Cardiology (JACC), o estudo que avaliou se as características da placa coronariana pela angiotomografia e a progressão da doença predizem a probabilidade de síndrome coronariana aguda no médio prazo. A presença de placa de alto risco (remodelamento positivo, hipoatenuação de placa e capa fibrótica fina) assim como a identificação de estenose significativa (redução luminal $>70 \%$ ) foram avaliadas em 3.158 pacientes, que foram classificados em 5 grupos: presença de placa de alto risco e de estenose; presença de placa de alto risco e ausência de estenose; ausência de placa de alto risco e presença de estenose; ausência de placa de alto risco e de estenose; e nenhuma placa; com taxas de eventos de $18,8 \%, 14,9 \%, 2,6 \%, 1,2 \%$ e $0,6 \%$, respectivamente. As taxas de eventos foram significativamente diferentes entre esses 5 grupos ( $p$ $<0,0001)$. Portanto, presença de sinais tomográficos de instabilidade de placa assim como a estenose coronariana são fatores de risco independentes para a ocorrência de síndrome coronariana aguda.

A AngioTC permite o diagnóstico da doença coronariana no estágio inicial, com a identificação da placa rica em lipídios. Essa análise até o momento é uma característica específica da AngioTC como método diagnóstico não invasivo.

\section{Acurácia e Confiabilidade Diagnóstica}

A acurácia da angiotomografia coronariana foi validada em 2008 com a publicação de três grandes estudos clínicos controlados (ACCURACY, CORE 64 e Meijboom WB, et al.). ${ }^{3-5}$ Estes estudos compararam a sensibilidade, especificidade e acurácia diagnóstica da angiotomografia coronariana com a angiografia invasiva em mais de 1.000 pacientes de risco baixo a intermediário para doença arterial coronariana. $\mathrm{Na}$ análise por paciente, a angiotomografia coronariana identificou a estenose igual ou maior que $50 \%$ com alta sensibilidade $(85 \%$ a $99 \%$ ) e alto valor preditivo negativo, variando entre $83 \%$ e $99 \%$ (Tabela 1 ).

Figura 1. Evolução do vaso normal até a formação da placa obstrutiva. A AngioTC de coronárias tem a capacidade de avaliação parietal, identificando precocemente a formação de doença coronariana.

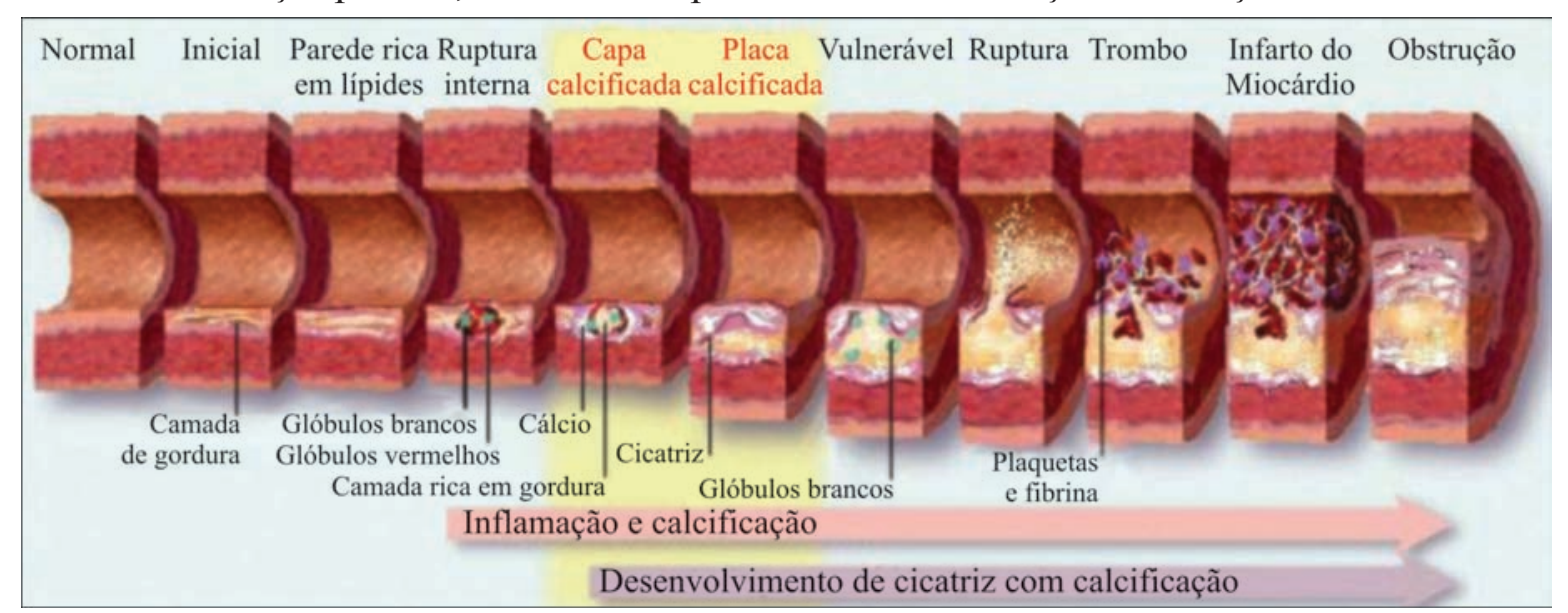


Figura 2. Placa não calcificada e com remodelamento positivo e núcleo hipodenso, na artéria descendente anterior (ADA) de um paciente jovem. Imagem MPR longitudinal (esquerda) e corte transversal da ADA no ponto de estenose (direita).
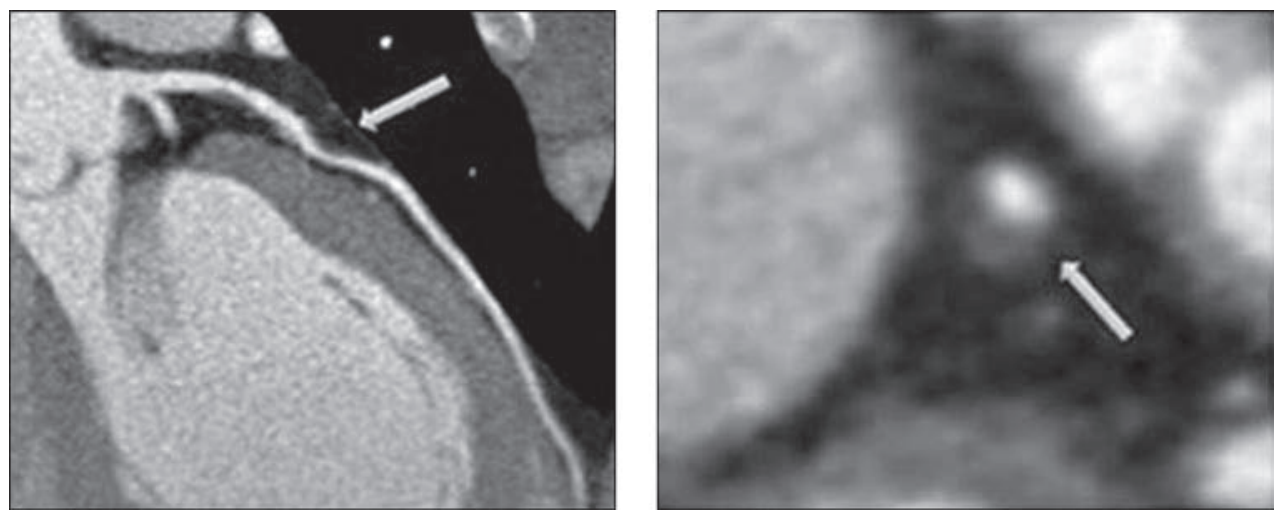

Figura 3. Placa não calcificada ocasionando estenose importante no segmento proximal da artéria descendente anterior (ADA) identificada pelaAngioTC (Figura 2A) e pela angiografia invasiva (Figura 2B).

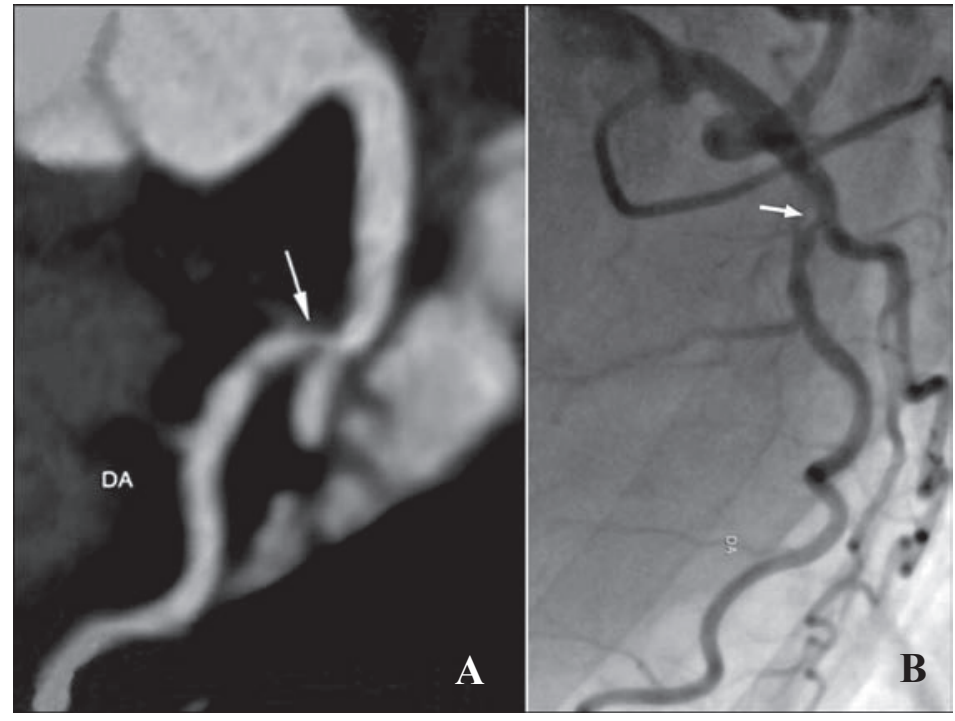

Budoff MJ e colaboradores ${ }^{6}$ publicaram em 2016, no JACC, uma metanálise que avaliou a angiotomografia como preditor de evento isquêmico na presença de lesões intermediárias a severas, tendo comparado diretamente com a angiografia coronariana invasiva $(\mathrm{ACI})$, associado à reserva de fluxo fracionada (FFR) como referência padrão. De acordo com o FFR, a isquemia estava presente em 151 (37\%) das 407 lesões. A precisão do diagnóstico, sensibilidade, especificidade, valor preditivo positivo e valor preditivo negativo foram $69 \%, 79 \%, 63 \%, 55 \%$ e $83 \%$ para a AngioTC; e $71 \%, 74 \%, 70 \%, 59 \%$ e $82 \%$ para angiografia invasiva. AUC para identificação de lesões causadoras de isquemia foi semelhante: 0,75 para AngioTC coronariana e 0,77 para CATE $(p=0,6)$. Portanto, pode-se concluir que a angiotomografia de coronárias fornece similar precisão diagnóstica para detecção e exclusão de lesão isquêmica quando comparado ao cateterismo, possuindo potencial para substituir em grande parte o CATE no diagnóstico de doença arterial coronariana obstrutiva em pacientes com probabilidade baixa e intermediária.

A seleção da técnica diagnóstica para detectar ou descartar doença arterial coronariana para um determinado paciente deve ser baseada na faixa ótima de probabilidade pré-teste do exame escolhido. Com 
Tabela 1. Acurácia diagnóstica da angiotomografia coronária.

\begin{tabular}{lcccccccccccc}
\hline & N & P & I & DAC & A & ASC & SENS & ESP & VPP & VPN & Falso + & Falso- \\
\hline ACCURACY & 230 & $25 \%$ & Não & Não & $88 \%$ & $88 \%$ & $95 \%$ & $83 \%$ & $64 \%$ & $99 \%$ & $17 \%$ & $5 \%$ \\
CORE 64 & 291 & $55 \%$ & Não & Sim & $66 \%$ & $86 \%$ & $85 \%$ & $90 \%$ & $91 \%$ & $83 \%$ & $9,4 \%$ & $15 \%$ \\
Meijboom et al. & 360 & $68 \%$ & Sim & Não & $88 \%$ & $88 \%$ & $99 \%$ & $64 \%$ & $86 \%$ & $97 \%$ & $35,6 \%$ & $1 \%$ \\
\hline
\end{tabular}

DAC - doença aterosclerótica coronária; SENS - sensibilidade; ESP - especificidade; VPP - valor preditivo positivo; VPN valor preditivo negativo; ASC - área sob a curva ROC; P - prevalência; A - acurácia; I - instável.

o intuito de avaliar o desempenho de testes não invasivos para determinar e descartar estenose coronariana significativa em pacientes com angina estável, foi publicada importante metanálise em 2018 por Knuuti J e colaboradores ${ }^{7}$. O objetivo foi determinar as faixas de probabilidade pré-teste (PPT) da doença arterial coronariana nas quais o teste de esforço, o ecocardiograma sob estresse, angiotomografia computadorizada coronariana, o SPECT, a tomografia por emissão de pósitrons (PET) e a ressonância magnética cardíaca (RMC) podem reclassificar a probabilidade pós-teste para definir ( $>85 \%)$ ou excluir $(<15 \%)$ anatomicamente (definida por avaliação visual de angiografia coronária invasiva) e funcionalmente $(\mathrm{FFR} \leq 0,8)$ a doença arterial coronariana significativa. $\mathrm{O}$ teste de esforço pode determinar e descartar a doença arterial anatomicamente significativa somente quando a PPT é $\geq 80 \%$ (76-83) e $\leq 19 \%$ (15-25), respectivamente. A angiotomografia computadorizada coronariana é capaz de determinar DAC com o valor de PPT $\geq$ $58 \%$ (45-70) e descartar quando a PPT $\leq 80 \%$ (6594). Os valores correspondentes de PPT para DAC funcionalmente significativa foram $\geq 75 \%(67-83) \mathrm{e}$ $\leq 57 \%$ (40-72) para angiotomografia de artérias coronárias e $\geq 71 \%$ (59-81) e $\leq 27 \%$ (24-31) para angiografia, demonstrando pior desempenho de imagens anatômicas contra FFR. Em contraste, as técnicas de imagem funcional (PET, estresse CMR e SPECT) são capazes de determinar doença arterial coronária funcionalmente significativa quando a PPT é $\geq 46-59 \%$ e descartar quando o PPT é $\leq 34-57 \%$. Portanto, a angiotomografia coronária apresenta-se com importante poder de exclusão de doença e relevante capacidade em definir DAC, destacandose dentre os outros métodos não invasivos.

\section{Prognóstico}

Nos últimos anos, vários trabalhos pequenos sugeriram a capacidade da angiotomografia de coronárias em fornecer importantes informações prognósticas, no entanto, só a partir de 2011 com a publicação do estudo $\mathrm{CONFIRM}^{8}$ que essa ideia foi validada.

O estudo CONFIRM ${ }^{8}$, publicado no Circulation, avaliou se o grau de obstrução das coronárias e a fração de ejeção (FE) eram fatores prognósticos independentes para a ocorrência de evento coronariano. Foram incluídos 14.064 pacientes, que foram categorizados como ausência de DAC, DAC não obstrutiva, obstrução de risco não alto (estenose $>50 \%$ em vaso único) e DAC de alto risco (TCE $>$ $50 \%$ ou doença multiarterial). A taxa de sobrevida livre de eventos foi maior no grupo sem DAC e menor no grupo com maior severidade da DAC, com significância estatística. Os resultados demonstraram que a severidade da DAC e a FEVE são fatores prognósticos independentes e que a obstrução coronariana tem valor incremental à $\mathrm{FE}$ associada às variáveis clínicas para predizer mortalidade por qualquer causa.

A consagração do papel prognóstico da angiotomografiade coronárias só ocorreu em 2015 com a publicação de dois grandes trabalhos: o estudo norte-americano PROMISE ${ }^{9}$ e o europeu SCOTHEART $^{10}$. Estes estudos comparam o papel da AngioTC com diferentes métodos funcionais na avaliação de pacientes com dor torácica estável e a ocorrência de desfechos clínicos.

O PROMISE 9 , estudo multicêntrico publicado no NEJM, apresentou as estratégias anatômica e funcional que foram usadas para diagnosticar DAC 
e avaliar sua severidade, assim como avaliar a FEVE da amostra estudada. Foram avaliados 10.003 pacientes randomizados em 2 grupos: investigação usual (teste ergométrico ou cintilografia miocárdica ou eco stress) e o grupo submetido a angiotomografia de artérias coronárias. O desfecho primário foi composto por morte por qualquer causa, infarto agudo do miocárdio (IAM), internações por síndrome coronariana aguda e complicações maiores relacionadas ao procedimento, não apresentando diferença estatística entre os grupos ao longo do seguimento. Mais pacientes no grupo da AngioTC foram submetidos ao CATE do que no grupo de investigação usual, entretanto, a proporção de cateterismos normais em 90 dias foi inferior no grupo da AngioTC (6,2\% vs 3,2\%).Foi indicada revascularização em 90 dias em 311 de 4.996 pacientes $(6,2 \%)$ no grupo AngioTC comparado com 158 de 5.007 (3,2\%) no grupo funcional $(p<0,001)$, incluindo cirurgia de revascularização miocárdica. Os resultados mostram, portanto, que o uso da angiotomografia das artérias coronárias como estratégia inicial em pacientes sintomáticos com suspeita de DAC, em comparação com testes funcionais, não apresentou melhores desfechos clínicos em um período de 2 anos.

O estudo SCOT-HEART ${ }^{10}$, publicado no JACC em 2015, envolveu 4.146 pacientes com dor torácica de baixo e moderado risco para DAC estável, que foram randomizados para investigação padrão (testes funcionais, sendo o teste ergométrico realizado em $85 \%$ dos casos) x investigação padrão associado à AngioTC de coronárias. $\mathrm{O}$ desfecho primário foi avaliar a ocorrência de morte e IAM não fatal em um seguimento de 20 meses. Quanto ao tratamento com medicações preventivas como AAS e estatina, o grupo AngioTC iniciou ou suspendeu em maior número o uso de medicações preventivas (AAS, estatina, IECA) quando comparado ao grupo investigação padrão.Possivelmente isso deveu-se à capacidade da angiotomografia em identificar mais DAC obstrutiva e não bstrutiva, proporcionando mudança no tratamento preventivo.AAngioTC foi associada com redução de $38 \%$ no risco de morte e IAM não-fatal, mas com pequena significância estatística (HR ajustado 0,62 IC 95\%, p=0,0527).
Enquanto a curva de Kaplan Meier para desfechos clínicos parece sugerir que as alterações no manejo de pacientes submetidos à AngioTC podem ter demorado mais de 6 semanas para serem efetivamente implementadas em alguns pacientes. Este estudo permite determinar que aAngioTC esclarece o diagnóstico de DAC e leva a alterações importantes nas investigações e tratamentos, implicando possivelmente em redução de eventos coronários fatais e não fatais.

Em 2016, foi publicada uma nova análise do estudo SCOT-HEART ${ }^{11}$ no JACC e trouxe como resultado que o grupo submetido à AngioTC apresentou maior frequência do uso de AAS e estatina quando comparado ao grupo investigação padrão, com significância estatística. Embora não tenha ocorrido redução no desfecho morte e IAM não fatal com significância estatística, as curvas do evento parecem sobrepor-se nos primeiros 50 dias e depois passam a se afastar. Esse achado não é considerado surpreendente já que nesse estudo houve claramente uma demora para a implementação de nova terapia após a realização da angiotomografia. Por isso, foram avaliadas as taxas de eventos após 50 dias da randomização, que foi o tempo mediano do início das terapias preventivas. Observou-se redução para metade das taxas de eventos fatais e IAM não fatal no grupo submetido a angiotomografia coronariana. Acredita-se que essa redução em eventos seja consistente com o tamanho do efeito atribuível ao início de terapias preventivas juntamente com potenciais benefícios de modificação do estilo de vida e revascularização coronária. Desse modo, conclui-se que a AngioTC leva ao uso mais apropriado da angiografia invasiva e a alterações em terapias preventivas, que foram associadas à redução para metade do infarto do miocárdio fatal e não fatal.

Um novo registro do CONFIRM $^{12}$ foi publicado em 2015 no AHA, buscando analisar especificamente o impacto do uso da estatina e aspirina na redução de morte coronariana em pacientes que após serem submetidos à angiotomografia receberam o diagnóstico de doença arterial coronária não obstrutiva (estenose $<50 \%$ ) ou ausência de DAC. Foi observado que terapia com estatina parece estar associada com redução significativa na mortalidade 
para os indivíduos com DAC não obstrutiva, não sendo observado este resultado na população sem DAC. Entretanto, o uso de AAS não implicou em redução da mortalidade em nenhum dos grupos.

Uma meta-análise ${ }^{13}$, publicada no Circulation em 2016, analisou a taxa de IAM e mortalidade por todas as causas em pacientes submetidos à angiotomografia de coronárias ou testes funcionais como estratégia diagnóstica.Houve uma tendência à maior realização de angiografias e revascularização entre pacientes submetidos à angiotomografia, o que foi atribuído aos achados do estudo Scot-Heart.Em comparação aos testes funcionais, a investigação inicial de suspeita de doença arterial coronariana estável usando a AngioTC resultou em redução significativa de IAM, aumento da incidência de revascularização coronariana e nenhum efeito na mortalidade por todas as causas.

Foi publicado em 2017 no JACC o estudo de Jørgensen ME e colaboradores ${ }^{14}$, que analisou a associação dos testes cardíacos não invasivos em pacientes com DAC estável e o uso subsequente de medicamentos, procedimentos invasivos e desfechos clínicos. Um total de 86.705 pacientes foram selecionados. Destes, 53.744 foram submetidos a testes funcionais e 32.961 a AngioTC. Foram seguidos por uma mediana de 3,6 anos. O estudo revela que pacientes estáveis submetidos à avaliação inicial de suspeita de DAC, a angiotomografia de coronárias foi associada a maior uso de estatinas, aspirina e procedimentos invasivos, assim como, custos mais elevados quando comparados com os testes funcionais (Tabela 2). A angiotomografia foi associada a uma menor taxa de IAM assim como desfecho combinado por IAM e mortalidade por todas as causas.

Em 2018, foi publicada a análise do SCOT HEART após média de 5 anos de seguimento. ${ }^{15}$ Este estudo envolveu 4.146 pacientes com dor torácica estável, encaminhados para tratamento padrão (testes funcionais), associado à realização de angiotomografia de artérias coronárias (2.073 pacientes) ou para o tratamento padrão isolado (2.073 pacientes). O desfecho primário foi morte por causas cardíacas ou infarto do miocárdio não fatal aos 5 anos de seguimento. A taxa do desfecho primário foi menor no grupo angiotomografia do que no grupo controle $(2,3 \%$
Tabela 2. Angiotomografia de coronárias versus testes funcionais.

\begin{tabular}{lccc}
\hline & AngioTC & $\begin{array}{c}\text { Testes } \\
\text { Funcionais }\end{array}$ & Valor p \\
\hline Estatina & $15,9 \%$ & $9,1 \%$ & $<0,001$ \\
AAS & $12,7 \%$ & $8,5 \%$ & $<0,001$ \\
CATE & $14,7 \%$ & $10,1 \%$ & $<0,001$ \\
ICP & $3,8 \%$ & $2,1 \%$ & $<0,001$ \\
Custos & US \$ 995 & US \$ 718 & $<0,001$ \\
\hline
\end{tabular}

vs 3,9\%; razão de risco, 0,59; Intervalo de confiança de 95\% [IC], 0,41 a 0,84; p =0,004). Apesar de nos primeiros meses de acompanhamento a quantidade de ACI e revascularização coronariana terem sido maiores no grupo de angiotomografia quando comparado com o grupo referência padrão, a taxa de realização de procedimento invasivo torna-se semelhante entre os grupos aos 5 anos. AACI foi realizada em 491 pacientes no grupo AngioTC e em 502 pacientes no grupo controle (razão de risco, 1,00; IC95\%, 0,88-1,13), enquanto a revascularização coronariana foi realizada em 279 pacientes no grupo AngioTC e em 267 no grupo controle (razão de risco, 1,07; IC 95\%, 0,91 -1,27). Nota-se, entretanto, que mais terapias preventivas foram iniciadas em pacientes do grupo AngioTC (odds ratio, 1,40; IC95\%, 1,191,65 ), assim como mais terapias antianginosas (odds ratio, 1,27; 95\% $\mathrm{IC}, 1,05-1,54)$. Não houve diferenças significativas entre os grupos nas taxas de morte cardiovasculares ou morte por qualquer causa. Portanto, o uso de angiotomografia em adição à realização de testes funcionais em pacientes com dor torácica resultou em uma taxa significativamente menor de morte por doença coronariana e infarto do miocárdio não fatal em 5 anos quando comparado ao tratamento padrão isoladamente, sem resultar em realização significativamente maior de angiografia coronariana ou revascularização coronariana.

\section{Avaliação Funcional}

As técnicas emergentes de perfusão por tomografia computadorizada miocárdica (CTP miocárdica) e reserva de fluxo fracionada guiada por tomografia computadorizada (FFR-CT) 
estabeleceram que a angiotomografia cardíaca é capaz de diagnosticar isquemia miocárdica com alta precisão diagnóstica quando comparada com vários padrões de referência, como perfusão miocárdica nuclear, imagem de perfusão miocárdica por ressonância magnética, angiografia invasiva e análise invasiva de reserva de fluxo fracionado (FFR).

O estudo CORE320 ${ }^{16}$, publicado em 2013, avaliou como objetivo primário a acurácia da perfusão miocárdica pela tomografia integrada com os resultados da angiotomografia, em comparação com a combinação dos resultados da ACI e a cintilografia miocárdica de perfusão em estresse (CMPE), considerada positiva a combinação de estenose $\geq 50 \%$ com déficit perfusional associado em território equivalente. E como desfecho secundário avaliou a acurácia da angiotomografia em relação à combinação de angiografia e CMPE. Para a combinação de estenose $\geq 50 \%$, detectada pela AngioTC e déficit de perfusão pela CTP, a sensibilidade, especificidade, valores preditivos positivos e predicativos negativos (IC 95\%) foram $80 \%$ (72-86), 74\% (68-80), 65\% (58-72) e $86 \%$ (80-90), respectivamente. Para a doença com déficit de perfusão definida pelo ACI-SPECT / MPI, a precisão da AngioTC foi significativamente aumentada pela adição de CTP na avaliação por paciente e por vaso. Portanto, esse estudo traz a informação de que a estratégia da adição de CTP à angiotomografia de coronárias permite identificar corretamente os pacientes com DAC obstrutiva (estenose $\geq 50 \%$ ) associada à alteração isquêmica.

O NXT trial ${ }^{17}$, publicado em 2014 no JACC, avaliou o desempenho diagnóstico da reserva de fluxo fracionada não invasiva (FFRCT), considerando a FFR invasiva (FFR-ICA) o método padrão ouro para definir a indicação de revascularização em pacientes com DAC estável. Foram incluídos 254 pacientes com placas com estenose $>50 \%$, sendo isquemia definida como FFRCT ou FFR $\leq 0,8$. A área sob a curva ROC para FFRCT foi de 0,90 (intervalo de confiança de 95\% [IC]: 0,87-0,94) vs 0,81 (IC 95\%: 0,76-0,87) para AngioTC coronariana $(p=0,0008)$. Sensibilidade e especificidade por paciente (IC 95\%) para identificar isquemia miocárdica foram $86 \%$ (IC 95\%: 77\%-92\%) e 79\% (IC 95\%: 72\%-84\%) para
FFRCT vs 94\% (86-97) e 34\% (IC 95\%: 27\%$41 \%$ ) para AngioTC coronariana e 64\% (IC 95\%: 53\%-74\%) e $83 \%$ (95\% CI: 77\%-88\%) para ACI, respectivamente. Em pacientes $(n=235)$, com estenose intermediária (IC 95\%: 30\%-70\%), a acurácia diagnóstica do FFRCT permaneceu alta.

Esse estudo evidencia que a FFRCT apresenta excelente acurácia, além de alta especificidade quando comparada com a AngioTC de coronárias isoladamente.

Em 2019, foi publicada uma subanálise do estudo PACIFIC $^{18}$, que avaliou, em pacientes com DAC estável, o desempenho do FFRCT para o diagnóstico de isquemia, quando comparado com a angiotomografia coronariana, SPECT e PET. Os valores da precisão diagnóstica,sensibilidade e especificidade da FFRCT foram 87\%, 90\% e 86\% por vaso e $78 \%, 96 \%$ e $63 \%$ por paciente, respectivamente. ASC para identificação de lesões causadoras de isquemia foi significativamente maior para FFRCT $(0,94$ e 0,92$)$ em comparação com a AngioTC coronariana $(0,83$ e 0,$81 ; p<0,01$ para ambos) e SPECT (0,70 e 0,75; $<<0,01$ para ambos), na avaliação por vaso e por paciente, respectivamente. FFRCT também apresentou melhor performance em determinar isquemia quando comparado ao PET na análise por vaso (AUC 0,87; $\mathrm{p}<0,01$ ), mas não na análise por paciente (AUC $0,91 ; \mathrm{p}=0,56)$.

\section{Mudança em Diretrizes e Guidelines}

O National Institute for Health and Care Excellences (NICE) Clinical Guideline em 2016 traz como recomendação a realização da angitomografia de artérias coronárias como estratégia de primeira linha em todos os pacientes que apresentaram dor torácica com a suspeita de doença arterial coronariana. ${ }^{19}$

\section{Conclusão}

Estudos robustos evidenciam o excepcional poder diagnóstico e valor prognóstico em indivíduos de probabilidade intermediária de DAC, sendo, portanto, uma modalidade de imagem não invasiva 
altamente eficaz para excluir ou detectar a doença coronariana obstrutiva em pacientes sintomáticos. Ademais, é razoável dizer que estudos corroboram o fato de que a adição da angiotomografia à estratégia padrão reduz eventos coronarianos, principalmente infarto não fatal.

\section{Referências}

1. Sekhri N, Feder GS, Junghans C, Hemingway H, Timmis AD. How effective are rapid access chest pain clinics? Prognosis of incident angina and non-cardiac chest pain in 8762 consecutive patients. Heart 2007;93:458-63.

2. Motoyama S, Ito H, Sarai M, et al. Plaque characterization by coronary computed tomography angiography and the likelihood of acute coronary events in mid-term follow-up. J Am Coll Cardiol. 2015;66:337-46.

3. BudoffMJ, Dowe D, Jollis JG, Gitter M, Sutherland J, Halamert E, et al. Diagnostic performance of 64- multidetector row coronary computed tomographic angiography for evaluation of coronary artery stenosis in individuals without known coronary artery disease: results from the prospective multicenter ACCURACY (Assessment by Coronary Computed Tomographic Angiography of Individuals Undergoing Invasive Coronary Angiography) trial. J Am Coll Cardiol. 2008;52:1724-32.

4. Miller JM, Rochitte CE, Dewey M, Arbab-Zadeh A, Niinuma H, Gottlieb I, et al. Diagnostic performance of coronary angiography by 64-row CT. N Engl J Med. 2008;359: 2324-36.

5. Meijboom WB, Meijs MF, Schuijf JD, Cramer MJ, Mollet NR, van Mieghem CA, et al. Diagnostic accuracy of 64slice computed tomography coronary angiography: a prospective, multicenter, multivendor study. J Am Coll Cardiol. 2008;52:2135-44.

6. Budoff MJ, Nakazato R, Mancini GB, et al. CT angiography for the prediction of hemodynamic significance in intermediate and severe lesions: head-to-head comparison with quantitative coronary angiography using fractional flow reserve as the reference standard. J Am Coll Cardiol Img. 2016;9:559-64.

7. Knuuti Saraste A, et al. The performance of non- invasive tests to rule-in and rule-out significant coronary artery stenosis in patients with stable angina: a meta-analysis focused on post-test disease probability. Eur Heart J. 2018;39:3233-330.

8. Chow BJ1, Small G, Yam Y, Chen L, Achenbach S, AlMallah M, et al. Incremental prognostic value of cardiac computed tomography in coronary artery disease using CONFIRM:COroNary computed tomography angiography evaluation for cli-nical outcomes: an InteRnational Multicenter registry. Circ Cardiovasc Imaging. 2011 Sep;4(5):463-72.
9. Douglas PS, Hoffmann U, Patel MR, Mark DB, Al-Khalidi HR, Cavanaugh B, et al. Outcomes of anatomical versus functional tes-ting for coronary artery disease. $\mathrm{N}$ Engl $\mathrm{J}$ Med. 2015 Apr 2;372(14):1291-300.

10. SCOT-HEART investigators. CT coronary an-giography in patients with suspected angina due to coronary heart disease (SCOT-HEART): an open-label, parallel-group, multicentre trial. Lancet 2015 Jun 13;385(9985):2383-91.

11. Williams MC, Hunter A, Shah AS, et al., for the SCOTHEART Investigators. Use of coronary computed tomographic angiography to guide management of patients with coronary disease J Am Coll Cardiol. 2016;67:1759-68.

12. Cho I, Chang HJ, B OH, et al. Incremental prognosticutilityofcoronary $\mathrm{CT}$ angiography for asymptomatic patients based upon extent and severity of coronary arterycalcium: results from the Coronary CT Angiography Evaluation For Clinical Outcomes International Multicenter (CONFIRM) study. Eur Heart J. 2015;36(8):501-8.

13. Bittencourt MS, Hulten EA, Murthy VL, et al. Clinical outcomes after evaluation of stable chest PERSPECTIVES pain by coronary computed tomographic angiography versus usual care: a metaanalysis. Cir Cardiovasc Imaging 2016;9:e004419.

14. Jørgensen ME, Andersson C, Nørgaard BL, Abdulla J, Shreibati JB, Torp-Pedersen C, et al. Functional testing or coronary computed tomography angiography in patients with sta- ble coronary artery disease. J Am Coll Cardiol. 2017;69:1761-70. doi:10.1016/ j.jacc.2017.01.046.

15. SCOT-HEART Investigators, Newby DE, Adamson PD, et al. Coronary CT Angiography and 5-Year Risk of Myocardial Infarction. N Engl J Med. 2018; 379:924.

16. Rochitte CE, George RT, Chen MY, et al. Com-puted tomography angiography and perfusion to as-sess coronary artery stenosis causing perfusion de-fects by single photon emission computed tomography: the CORE320 study. Eur Heart J. 2014;35:1120-30.

17. Norgaard BL, et al. Diagnostic performance of noninvasive fractional flow reserve derived from coronary computed tomography angiography in suspected coronary artery disease: the NXT trial (analysis of coronary blood flow using CT angiography: next steps). J Am Coll Cardiol. 2014;63:1145-55.

18. Driessen RS, Danad I, Stuijfzand WJ, et al. Comparison of coronary computed tomography angiography, fractional flow reserve, and perfu- sion imaging for ischemia diagnosis. J Am Coll Cardiol. 2019;73:161-73.

19. National Institute for Health and Care Excellence. Chest pain of recent onset: assessment and diagnosis of recent onset chest pain or discomfort of suspected cardiac origin (update). Clinical Guideline 95. London: NICE, 2016. 